\title{
Acta
Biochimica
Polonica
}

Vol. 51 No. 1/2004

$115-119$

QUARTERLY

\section{Biological evaluation of analogues of an insect neuropeptide proctolin}

\author{
Iwona Woźnica ${ }^{1}$, Wioletta Szeszel-Fedorowicz ${ }^{1}$, Grzegorz Rosiński ${ }^{2}$ \\ and Danuta Konopińska ${ }^{1 凶}$ \\ ${ }^{1}$ Faculty of Chemistry, University of Wroctaw, Wroctaw, Poland; ${ }^{2}$ Department of Animal \\ Physiology, A. Mickiewicz University, Poznań, Poland
}

Received: 31 October, 2003; revised: 23 January, 2004; accepted: 06 February, 2004

Key words: proctolin, insect myotropic peptide proctolin, proctolin analogues

Continuing our studies on proctolin (Arg-Tyr-Leu-Pro-Thr) we performed the synthesis and biological evaluation of 52 analogues substituted in position 2, 3, 4, and 5 of the peptide chain. The peptides were bioassayed for cardiotropic activity in vitro on Tenebrio molitor and myotropic activity on foregut of Schistocerca gregaria. Twenty analogues retained $20-80 \%$ of proctolin activity.

Continuing our studies on structure/function relationship of proctolin ( Konopińska \& Rosiński, 1999) (Arg-Tyr-Leu-Pro-Thr) we performed the synthesis of four groups of analogues modified in position $2,3,4$, or 5 by nat- ural or non-natural amino-acid residues, such as: 1/ H-Arg- $\mathrm{X}^{2}$-Leu-Pro-Thr-OH, where $\mathrm{X}^{2}=$ Hpa (1), $\mathrm{Hpa}\left(4-\mathrm{NO}_{2}\right)$ (2), $\mathrm{Hpa}\left(4-\mathrm{NH}_{2}\right)$ (3), $\mathrm{Hpa}\left(4-\mathrm{N}, \mathrm{N}-\mathrm{Me}_{2}\right.$ ) (4), Phg (5), D-Phg (6), Phg(4- $\left.\mathrm{NO}_{2}\right)$ (7), D-Phg(4-NO $\left.{ }_{2}\right)(8), \mathrm{Phg}\left(4-\mathrm{NH}_{2}\right)$

\footnotetext{
${ }^{凶}$ To whom correspondence should be addressed: Danuta Konopińska, Faculty of Chemistry, University of Wrocław, 50-383 Wrocław, Poland; e-mail: dk@wchuwr.chem.uni.wroc.pl

Abbreviations: The symbols of amino acids, peptides, and their derivatives are in accordance with the IUPAC-IUB Joint Commission on Biochemical Nomenclature Recommendation, 1983 (Eur J Biochem.; 138: 9, (1984); and J Pept Sci. 5: 465 (1999); J Pept Sci. 9: 1 (2003)).

$\gamma$-Abu, $\gamma$-aminobutyric acid; Boc-, $t$-butoxycarbonyl; BOP, benzotriazolyl- $N$-oxytrisdimethylaminophosphonium hexafluorophosphate; HOBt, hydroxybenztriazole; HPLC, high pressure liquid chromatography; DCC, $N, N$-dicyclohexylcarbodiimide; Hpa, L-homo-phenylalanine; Hpa(p- $\left.\mathrm{NO}_{2}\right)$, 4-nitro-L-homo-phenylalanine; $\mathrm{Hpa}\left(\mathrm{p}-\mathrm{NH}_{2}\right)$, 4-amino-L-homo-phenylalanine; $\mathrm{Hpa}\left(\mathrm{NMe}_{2}\right), 4$ $p$-N,N-dimethyl-L-phenylglycine; Me, methyl; Nva, L-norvaline; Phg, L-phenylglycine; Sar, sarcosine; Phg(p-OH), 4-hydroxy-L-phenylglycine; D-Phg(p-OH), 4-hydroxy-D-phenylglycine; Phg(p- $\left.\mathrm{NO}_{2}\right)$, 4-nitro-L-phenylglycine; D-Phg(p- $\left.\mathrm{NO}_{2}\right)$, 4-nitro-D-phenylglycine; $\mathrm{Phg}\left(\mathrm{p}-\mathrm{NH}_{2}\right), 4$-amino-L-phenylglycine; D-Phg(p- $\left.\mathrm{NH}_{2}\right)$, 4-amino-D-phenylglycine; $\mathrm{Phg}\left(\mathrm{p}-\mathrm{NMe}_{2}\right), 4-p-N, N$-dimethyl-L-phenylglycine; D-Phg(p- $\mathrm{NMe}_{2}$ ), 4-N,N-dimethyl-D-phenylglycine.
} 
(9), D-Phg(4- $\mathrm{NH}_{2}$ ) (10), Phg(4-OH) (11), D-Phg(4-OH) (12), Phg (4-N,N-Me 2 ) (13), D-Phg (4-N,N-Me ${ }_{2}$ (14); 2/H-Arg-Tyr-X ${ }^{3}$-ProThr-OH, where $\mathrm{X}^{3}=$ Ile (15), Phe (16), Lys (17), Glu (18), Gln (19), Asn (20), Asp (21), Nva (22), Nle (23), $\gamma$-Abu (24), Arg (25), Sar (26); 3/ H-Arg-Tyr-Leu- ${ }^{4}$-Thr-OH, where $\mathrm{X}^{4}=$ Phg (27), Phe (28), Ala(N-Me) (29); 4/ H-Arg-Tyr-Leu-Pro- $\mathrm{X}^{5}-\mathrm{OH}$, where $\mathrm{X}^{5}=\mathrm{Val}$ (30), D-Val (31), Ile (32), D-Ile (33), Ala (34), D-Ala (35), Ser (36), Asn (37), Gln (38), Asp (39), Glu (40), Arg (41), D-Arg (42), Lys (43), Gly (44); 5/ Arg-Tyr-Leu-Pro-NH( $\mathrm{R}^{\prime}$ ), where $\mathrm{R}^{\prime}$ = isobutyl (45), S-1-methyl-1-phenylmethyl (46), R-1-methyl-1-phenylmethyl (47), R-2-hydroxy-1-methylethyl (48), S-2-hydroxy1-methylethyl (49), R-2-hydroxypropyl (50), S-2-hydroxypropyl (51), 1-hydroxy-n-propyl (52) (Table 1). In the first group of analogues the Tyr residue in position 2 was replaced by L-Hpa and its derivatives or by L- and D-Phg derivatives. In contrast to native Tyr-2, Phg is an amino acid which is deprived of the methylene group between the $\mathrm{C}-\alpha$ atom and the benzene ring, whereas Hpa have two methylene groups between the $\mathrm{C}-\alpha$ atom and the benzene ring. The aim of these modifications was to explain the influence of lack of the methylene system or presence of an additional methylene group in the side chain of amino acids in position 2 on the myotropic activity in insects. Because the role of Leu in position 3 and Pro in position 4 has not been sufficiently established in the previous investigations (Konopińska \& Rosiński, 1999) of the structure-biological function relationship, we performed the synthesis of further proctolin analogues where Leu-3 and Pro-4 were replaced for protein or non-protein amino-acid residues. We also synthesized of proctolin analogues modified in position $5(30-52)$ to check the role of the C-terminal Thr on the biological activity in insects. In this group, Thr in position 5 was substituted with other amino acids. Especially interesting in these studies were analogues modified in position 5 by a residue with an isosteric structure of the side chain relative to Thr, such as $\left[\mathrm{Val}^{5}\right]$ - and $\left[\mathrm{Ile}^{5}\right]$-proctolin as well as analogues deprived of the carboxyl group at the C-terminal end of proctolin. In these analogues, $\mathrm{Thr}^{5}$ was replaced by a series of S- and R- hydroxyamines or phenyl amines. We also obtained analogues 50 and 51, with amines isosteric to the structure of the side chain of Thr in position 5 . The purpose of these studies was to explain the role of the C-terminal carboxyl group for proctolin myotropic properties in insects.

\section{MATERIALS AND METHODS}

Syntheses of the peptides were carried out by the solid-phase standard method (Kuczer et al., 1996). All peptides were synthesized by the classical solid-phase Boc (tert-butoxycarbonyl) procedure.

Chloromethylated classical Merrifield resin containing $0.7-0.8 \mathrm{mmol} \mathrm{Cl} / \mathrm{g}$ resin was obtained by the standard caesium salt procedure. The protected amino acid was coupled to the resin with diisopropylcarbodiimide or dicyclohexylcarbodiimide (DCC) in the presence of HOBt or BOP as coupling reagents. The Boc-protecting group was removed by $30 \% \mathrm{CF}_{3} \mathrm{COOH}$ in $\mathrm{CH}_{2} \mathrm{Cl}_{2}$. Peptides were released from the resin using $\mathrm{CF}_{3} \mathrm{SO}_{3} \mathrm{H}$, the nitro group was reduced by hydrogenation over $\mathrm{Pd} / \mathrm{C}$ as a catalyst. All free peptides were desalted with Amberlite CG-4B and then purified on a Sephadex G-15 column, with $5 \%$ acetic acid as eluent, according to (Kuczer et al., 1996). Final purification was carried out by preparative HPLC on a C-18 column (Beckman). Purity and homogeneity of the free peptides were determined by analytical HPLC, amino acid analysis, and optical activity. The purity of all peptides was about $100 \%$.

All peptides were bioassayed in vitro on semi-isolated hearts of yellow mealworm (Tenebrio molitor) using a cardioexcitatory test elaborated by Gäde and Rosiński (1988). Peptides 5-14 and 30-52 were bioassayed on the locust (Schistocerca gregaria) foregut 
(Gray et al., 1994). Results of biological investigation are presented in Table 1.

\section{RESULTS AND DISCUSSION}

Among the proctolin analogues modified in position 2 of the peptide chain studied for cardiotropic activity in T. molitor only peptides 1-4 retained weak $20 \%$ proctolin activity at the physiological range of concentrations $\left(10^{-9}-10^{-7} \mathrm{M}\right)$. Analogues 5-12 modified by D- or L-Phg and their derivatives were inactive. In the myotropic test performed on proctolin modified in position 2 of the peptide chain by L-homophenylalanine or its derivatives, there is a longer distance between the side chain aromatic ring at position 2 and the peptide chain. Thus, the presence of only one methylene group next to the benzene ring of the amino-acid residue at position 2 is essential for myotropic activity in insects. The myotropic effects observed in insects depend probably on the structural properties of the amino-acid residue at position 2 of the proctolin molecule. The biological activity of proctolin analogues modified at position 3 of the peptide chain tested (Table 1) on the

Table 1. Selected proctolin analogues with myotropic activity in insect

\begin{tabular}{|c|c|c|}
\hline \multirow[t]{2}{*}{ Peptide } & \multicolumn{2}{|c|}{$\begin{array}{l}\text { Myotropic effect of proctolin analogues } \\
\text { in comparison to native proctolin }(\%)\end{array}$} \\
\hline & $\begin{array}{c}\text { T. molitor heart } \\
\text { (at } 10^{-9}-10^{-7} \mathrm{M} \text { conc.) }\end{array}$ & $\begin{array}{l}\text { S. gregaria foregut } \\
\text { (at } 10^{-6} \mathrm{M} \text { conc.) }\end{array}$ \\
\hline H-Arg-Hpa-Leu-Pro-Thr-OH (1) & 20 & nd * \\
\hline H-Arg-Hpa(4- $\left.\mathrm{NO}_{2}\right)$-Leu-Pro-Thr-OH (2) & 20 & nd \\
\hline H-Arg-Hpa(4- $\mathrm{NH}_{2}$ )-Leu-Pro-Thr-OH (3) & 20 & nd \\
\hline H-Arg-Hpa(4-N,N-Me 2 )-Leu-Pro-Thr-OH (4) & 20 & nd \\
\hline H-Arg-Phg(4-N,N-Me ${ }_{2}$ )-Leu-Pro-Thr-OH (13) & inactive & 70 \\
\hline H-Arg-Tyr-Ile-Pro-Thr-OH (15) & 70 & nd \\
\hline H-Arg-Tyr-Lys-Pro-Thr-OH (17) & 65 & nd \\
\hline H-Arg-Tyr-Glu-Pro-Thr-OH (18) & 30 & nd \\
\hline H-Arg-Tyr-Asp-Pro-Thr-OH (21) & 70 & nd \\
\hline H-Arg-Tyr-Arg-Pro-Thr-OH (25) & 60 & nd \\
\hline H-Arg-Tyr-Leu-Pro-Val-OH (30) & 50 & 60 \\
\hline H-Arg-Tyr-Leu-Pro-Ile-OH (32) & 20 & 50 \\
\hline H-Arg-Tyr-Leu-Pro-D-Ile-OH (33) & inactive & 30 \\
\hline H-Arg-Tyr-Leu-Pro-Ala-OH (34) & 40 & inactive \\
\hline H-Arg-Tyr-Leu-Pro-Ser-OH (36) & 50 & inactive \\
\hline H-Arg-Tyr-Leu-Pro-Asn-OH (37) & 20 & inactive \\
\hline H-Arg-Tyr-Leu-Pro-Gln-OH (38) & inactive & 80 \\
\hline H-Arg-Tyr-Leu-Pro-Arg-OH (41) & 60 & inactive \\
\hline H-Arg-Tyr-Leu-Pro-Lys-OH (43) & 20 & inactive \\
\hline H-Arg-Tyr-Leu-Pro-(S)-NHCH${ }_{2} \mathrm{CH}(\mathrm{OH}) \mathrm{CH}_{3}(51)$ & 60 & nd \\
\hline H-Arg-Tyr-Leu-Pro- $\mathrm{NHCH}_{2} \mathrm{CH}_{2} \mathrm{CH}_{2} \mathrm{OH}$ (52) & 50 & nd \\
\hline Other peptides & inactive & inactive \\
\hline
\end{tabular}

*nd, no data

the foregut of $S$. gregaria, analogues modified in position 2 of the peptide chain (5-12) were inactive. The lack of myotropic activity of proctolin analogues containing L- or D-phenylglycine (5-12) is probably a result of the lack of the $-\mathrm{CH}_{2}-$ group in the side chain of the Phg residue. It is probably an important element of structure for creation of myotropic activity in insects. In the analogues of
T. molitor heart showed that the presence of hydrophobic residues in position 3 is not so important for myotropic properties in insects as we postulated in our earlier paper (Konopińska \& Rosiński, 1999). Only [Val $\left.{ }^{3}\right]$-proctolin, described earlier (Konopińska \& Rosiński, 1999), and [Ile ${ }^{3}$-proctolin (15) presented here both with a branched side chain, retain a high cardiostimulatory activity. 
Proctolin analogues containing hydrophobic amino acids without a branched side chain, such as: Phe (16), Sar (26), Nva (22), Nle (23), and $\gamma$-Abu (24) were practically inactive. On the other hand, proctolin analogues modified at position 3 by basic amino-acids, such as (17) and (25), retained more than $60 \%$ of proctolin activity. Analogues containing acidic amino-acid residues at position 3 , like Asp (21) or Glu (18), had 70\% and 30\% of the native peptide activity, respectively. The exchange of Thr for amines also pointed out that the presence of the C-terminal carboxyl group is important for the myotropic activity. Peptides 30, 32, 34, 41, 43, 51, and 52 retained about $20-60 \%$ of the proctolin activity in T. molitor. We found that analogues 33,30 and 32 retained about $30-60 \%$ of the proctolin-like activity in the myotropic test on the foregut of $S$. gregaria whereas peptides 34 and 36 had weak stimulatory properties in the test evaluating contraction of the locust foregut at the peptide concentration ranging from $10^{-7}$ to $10^{-6}$ M. Other peptides had neither agonistic nor antagonistic activity. This is interesting that analogues 30 and 32 , containing Val or Ile with the side chain isosteric in relation to Thr, stimulated the heart-beat frequency in $T$. molitor and contraction of the $S$. gregaria foregut. It testifies that the presence of methyl or ethyl groups isosteric with the threonine side chain is sufficient for preservation of the myotropic effect in insects. In addition, it should be pointed out that proctolin analogue 51, containing R-1-amino-2-propanol in position 5 , and analogue 52, containing 1-amino-n-propanol with the side chain isosteric with the side chain of Thr, retained about $50 \%$ of proctolin myotropic activity in T. molitor. Moreover, species specificity was observed in the case of [D-Ile ${ }^{5}$-(33) and $\left[G n^{5}\right]$-proctolin (38). These different myotropic effects, observed in two insect species, depend probably on the structural requirement for a specific amino-acid residue in position 5 of the proctolin molecule in the species tested. For instance, a peptide with Ala in po- sition 5 of the proctolin chain (analogue 34) weakly stimulated the heart of the yellow mealworm. The same analogue showed no stimulatory effect in the test performed on the foregut of $S$. gregaria. Similar species specificity was observed in the case of analogues 33 and 38, which contained D-Ile and Gln, respectively, instead of Thr-5. These analogues preserved $30 \%$ and $80 \%$ of the proctolin activity in the locust, respectively, while lacked this activity in the yellow mealworm. The different activities of the investigated peptides in the two insect species is probably a consequence of structurally different requirement of receptors in the T. molitor heart and S. gregaria foregut.

\section{CONCLUSION}

From analysis of the myotropic effects of proctolin analogues 1-52 in insects the following conclusions can be drawn:

1. The biological results pointed out that the presence of a methylene group between the C- $\alpha$ atom and the aromatic ring of the side chain of the amino-acid residue at position 2 of the peptide chain is important for myotropic activity in insects.

2. Probably, elongation of the side chain at position 2 and bringing the aromatic ring further away from the peptide chain changes the peptide conformation and leads to inactive analogues.

3. The presence of a hydrophobic amino-acid residue with a branched side chain at position 3 is important for cardiotropic activity in the yellow mealworm.

4. The basic or acidic character of residues at position 3 of proctolin does not destroy the myotropic activity on the insect heart.

5 . The lack of activity of most analogues containing amines instead of Thr in position 5, except for analogues 51 and 52 which retained $60-50 \%$ proctolin activity, testifies that the presence of the carboxyl group at the C-terminus as well as of the -OH group in the 
side chain plays an essential role for the myotropic properties in insects.

\section{R E F E R E N C E S}

Gray AS, Osborne RH, Jewess PJ. (1994) Pharmacology of proctolin receptors in the isolated foregut of the locust Schistocerca gregaria - identification of [ $N$-methyl-L-tyrosine ${ }^{2}$-proctolin as a potent receptor antagonist. J Insect Physiol.; 40: 595-600.
Konopińska D, Rosiński G. (1999) Proctolin an insect neuropeptide. J Pept Sci.; 5: 533-46.

Kuczer M, Rosiński G, Lisowski M, Picur B, Konopińska D. (1996) New proctolin analogues modified by D-amino acids in the peptide chain and their high cardioexcitatory effect on Tenebrio molitor. Int $J$ Peptide Protein Res.; 48: 289-91.

Rosiński G, Gäde G. (1988) Hyperglycaemic and myoactive factors in the corpora cardiaca of the mealworm Tenebrio molitor. J Insect Physiol.; 33: 451-63. 